\title{
The presence and clinical implications of $\alpha$-2,6-galactose-linked sialic acids in non-small-cell lung cancer brain metastases - preliminary study
}

\author{
Maciej J. Pelak ${ }^{1}$, Bozena Jarosz ${ }^{2}$, Anita Straczynska-Niemiec ${ }^{3}$, \\ Pawel Krawczyk $^{4}$, Piotr Skoczylas ${ }^{5}$, Katarzyna M. Pecka ${ }^{6}$, Miroslaw Snietura ${ }^{3}$, \\ Justyna Szumilo ${ }^{7}$, Tomasz Trojanowski ${ }^{2}$ \\ ${ }^{1}$ Department of Radiation Therapy, Maria Sklodowska-Curie Memorial Institute of Oncology \\ - Gliwice Branch, Poland \\ ${ }^{2}$ Department of Neurosurgery and Pediatric Neurosurgery, Medical University of Lublin, Poland \\ ${ }^{3}$ Department of Tumor Pathology, Maria Sklodowska-Curie Memorial Institute of Oncology \\ - Gliwice Branch, Poland \\ ${ }^{4}$ Department of Pneumology, Oncology and Allergology, Medical University of Lublin, Poland \\ ${ }^{5}$ Department of Thoracic Surgery, Medical University of Lublin, Poland \\ ${ }^{6}$ Department of Surgery, St Elisabeth Hospital, Katowice, Poland \\ ${ }^{7}$ Department of Pathomorphology, Medical University of Lublin, Poland
}

\begin{abstract}
Brain metastases (BM) in non-small-cell lung cancer (NSCLC) patients present an increasing clinical challenge. Identifying biomarkers which specifically identify patients at high risk of BM may improve their early diagnosis, which is crucial for surgical and radiotherapeutic treatment outcome. Alpha-2,6-sialyltransferase $(\alpha-2,6-\mathrm{ST})$ and the primary product of its activity, alpha-2,6-galactose-linked sialic acids $(\alpha-2,6-\mathrm{GalSA})$ have been found responsible for the adhesion of tumor cells to the brain vessels' endothelium and enabling their transmigration through the blood-brain barrier in brain metastatic tumors. The aim of the study was to investigate by histochemical method the presence and possible role of $\alpha-2,6-\mathrm{GalSA}$ in the formation of brain metastasis in NSCLC. In the screening phase 76 metastatic brain tumors were stained for $\alpha-2,6-\mathrm{GalSA}$ and the second phase involved an identical staining of 20 primary tumors of patients who had their primary tumors treated with surgery or definite radiochemotherapy yet who later developed BM. The results were compared to a control group of 22 patients treated with surgery for NSCLC and who survived 5 years without the recurrence of disease. Alpha-2,6-GalSA presence was found to be down-regulated in poorly differentiated tumor types, whereas majority of differentiated tumors overexpressed it. This was statistically significant for both BM and the primary tumors. The expression of $\alpha-2,6-\mathrm{GalSA}$ remained stable in primary and metastatic tumor pairs, however, no statistically significant differences were observed between study and control groups. Within the study group, a higher $\alpha$-2,6-GalSA expression was associated with better overall survival, but not all statistical models found this result significant. Further studies are recommended to validate these findings. (Folia Histochemica et Cytobiologica 2014, Vol. 52, No. 2, 104-111)
\end{abstract}

Key words: alpha-2,6-sialic acids; NSCLC; brain metastases; survival; blood-brain barrier; CAMs 


\section{Introduction}

Non-small-cell lung cancer (NSCLC) accounts for approximately $85 \%$ of cases of malignant lung tumors [1]. During its course an event of metastasis to the central nervous system (CNS) is always associated with a significantly worse survival prognosis of the patient. Due to recent advances in the treatment modalities of locally advanced or metastatic NSCLC such as chemotherapy, irradiation and molecularly targeted agents, the overall survival time of lung cancer patients has considerably extended. As a result the incidence of brain metastasis (BM) in NSCLC is also rising, reaching up to $40 \%$ in certain cohorts [2]. A precise identification of BM risk factors in NSCLC would allow for highlighting a group of patients who should be followed up routinely with high-sensitivity imaging modalities such as CT or MRI, even with no clinical symptoms present. Such patients would have a greater chance for earlier detection of brain metastasis and this is widely known to increase the efficiency of surgery and irradiation, the two basic treatment modalities [3]. As for the biological factors of risk, there are BM-focused studies investigating the following particles: epidermal growth factor receptor (EGFR), BAX, cyclooxygenase 2 (COX2), erbB2, factor VIII, ephrin type-A receptor 2 (EphA2), urokinase plasminogen activator (UPA), UPA receptor (UPAR), plasminogen activator inhibitor type 1 (PAI-1), matrix metalloproteinase type 9 (MMP9), tissue inhibitor of matrix metalloproteinases type 1 (TIMP1), p53 and e-cadherin [4-6].

Alpha-N-acetyl-neuraminyl-2,3-beta-galactosyl1,3-N-acetylgalactosaminide alpha-2,6-sialyltransferase 5 (further referred to as $\alpha$-2,6-sialyltransferase or $\alpha-2,6-\mathrm{ST})$ is a type II trans-membrane protein, encoded by ST6GalNAC5 gene (1p31.1, 198271 bp). Structurally, it is a 336 amino acids long, $38443 \mathrm{Da}$ enzyme that exists predominantly within the Golgi and trans-Golgi network of the cell. Its chemical function is to catalyze the transfer of sialic acid to glycolipids, predominantly to the terminal galactose by an $\alpha-2,6$ linkage. A few physiological roles have been assigned to the products of its activity, i.e. alpha-2,6-galactose-linked sialic acids $(\alpha-2,6-\mathrm{GalSA})$. Various studies have found $\alpha-2,6-$ GalSA to be associated with cell differentiation and proliferation [7], intercellular and cell-matrix interactions [8] and immunity [9]. Their role in cancer has not yet been fully established, although available studies tend to demonstrate it as an indicator of poor prognosis [10-12].

The main reason for $\alpha-2,6-\mathrm{ST}$ and $\alpha-2,6-\mathrm{GalSA}$ to be studied by oncologists was discovery of their potential role in the formation of brain metastasis.
The initial genome-wide association study found the ST6GalNAC5 gene to be exclusively overexpressed in brain metastatic tumors of breast cancer [13]. A histochemical staining for $\alpha-2,6$-GalSA confirmed their absence in both primary breast cancer tumors and other metastatic sites - including the liver, bones and lungs [13]. Alpha-2,6-ST and $\alpha-2,6-\mathrm{GalSA}$, the products of the enzyme's activity, may thus have a highly specific function which acquisition over time enables tumor cells to colonize the brain. That function was identified in an experimental model as to enable the adhesion of tumor cells to brain vasculature endothelium as well as increase the permeability of the blood-brain barrier (BBB) [13]. These findings, as well as initial reports on development of drugs targeting sialyltransferases [14] justified a preliminary study on the presence and possible clinical role of $\alpha-2,6-\mathrm{GalSA}$ as a product of $\alpha-2,6$-ST activity in NSCLC and its brain metastases, which has yet not been described.

\section{Material and methods}

Detection and quantification of $\alpha-2,6-$ GalSA expression. In order to detect the presence of $\alpha-2,6-\mathrm{GalSA}$ in tumor cells, a histochemical staining protocol described by de Bos at al. [13] was followed. The detection involved use of a specific lectin, the Sambucus nigra agglutinin (SNA) which binds with high affinity to the terminal sialic acid in an $\alpha-2,6$, and, to a lesser extent, in an $\alpha-2,3$, linkages [15]. On the basis of a study by Kaneko et al. [16] appropriate normal human tissues were selected for positive and negative controls - the choroid plexus and the small intestine, respectively (Figure 1). The staining protocol was established as following: representative paraffin-embedded tumor samples were first cut into $3 \mu \mathrm{m}$ thick slides. The endogenous peroxidase activity was blocked by immersion for $30 \mathrm{~min}$ in $3 \%$ hydrogen peroxide in methanol. Incubations in streptavidin and biotin solution (Vector Labs ${ }^{\mathrm{TM}}$, Burlingame, CA, USA; kit), 15 min each, were followed by two incubations (30 min each) with bovine serum albumin (BSA) blocking solution (Thermo Scientific, Waltham, MA, USA; diluted 1:1 in PBS) and biotinylated SNA lectin (Vector Labs, diluted 1:200 in PBS, resulting concentration $10 \mu \mathrm{g} / \mathrm{ml}$ ). After each subsequent incubation the reagents were washed with PBS + Tween solution as proposed by the manufacturer. Afterwards, streptavidin-horseradish peroxidase and diaminobenzidine (DAB) $-\mathrm{H}_{2} \mathrm{O}_{2}$ solutions (Vector Labs) were applied subsequently according to the manufacturer's instructions for 30 and 2 min, respectively. Finally, the sections were counterstained with hematoxylin for $30 \mathrm{sec}$ (possibility of visualizing interaction of hematoxylin and lectin was excluded in test series). Sections were then washed with distilled water and automatically covered using the Dako Toluene-Free Mounting Medium (Dako, Glostrup, Denmark). 

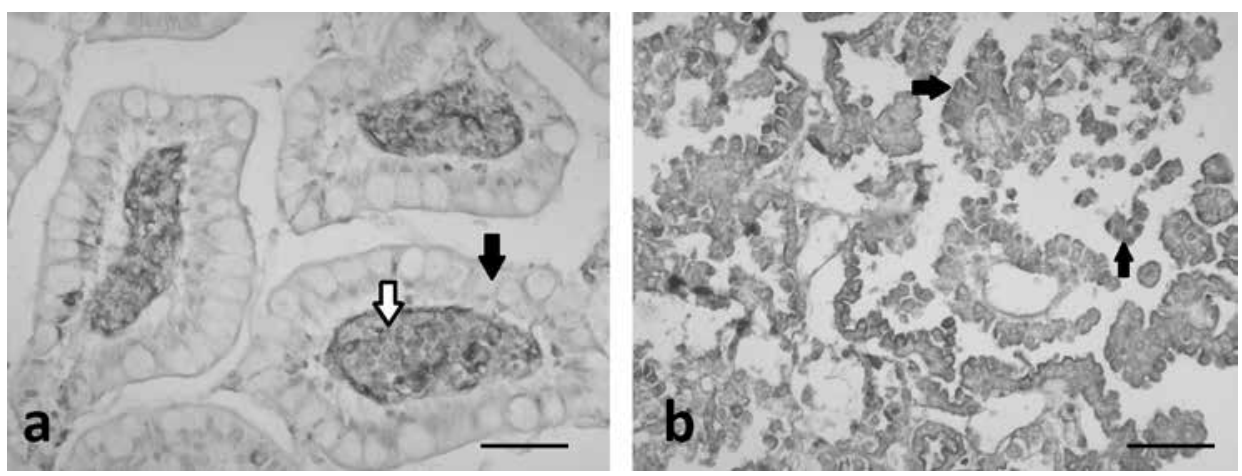

Figure 1. Reference histochemical staining of $\alpha$-2,6-galactose-linked sialic acids by Sambucus nigra agglutinin (SNA). A. Negative SNA staining of goblet cells of the small intestine (black arrow). Note the positive staining of the connective tissue underneath (white arrow). B. Strong positive SNA staining of the choroid plexus in all of the cells depicted (black arrows). Nuclei were counterstained with hematoxylin. Magnification $=\times 200$; bars, $50 \mu \mathrm{m}$

Table 1. Basic scoring system used for the evaluation of the number SNA-positive cells and intensity of $\alpha-2,6-\mathrm{GalSA}$ expression

\begin{tabular}{|l|c|c|c|}
\hline $\begin{array}{l}\text { \% of positive } \\
\text { cells }\end{array}$ & Score A & Staining intensity & Score B \\
\hline $0-10$ & 0 & $(-)$ & 0 \\
\hline $10-25$ & 1 & + & 1 \\
\hline $26-50$ & 2 & ++ & 2 \\
\hline $51-75$ & 3 & +++ & 3 \\
\hline $76-100$ & 4 & & \\
\hline
\end{tabular}

The level of staining of $\alpha$-2,6-GalSA was microscopically assessed blindly and independently by two experienced neuropathologists (B.J. and A.S-N.). We opted for the use of a modified Allred scoring system initially used for quantification of steroid receptors because it provides information on both number of the stained cells and the staining intensity, thus providing more data about the expression of studied compound in a tumor tissue $(\alpha-2,6-\mathrm{GalSA}$ in case of our study) [17]. The evaluation system for the percentage of cells which stain and the staining intensity are shown in Table 1. The total score, referred to as histochemical score (HC) has been established as a sum of A and B scores.

If the $\mathrm{HC}$ score for a particular tumor varied between the researchers (22 cases out of 118 in total), the average $\mathrm{HC}$ score was included into the results. We arbitrarily interpreted tumors which gained $\mathrm{HC}$ score of 6 or more as positive for $\alpha-2,6-$ GalSA staining.

Characteristics of patients and tumors. The study was divided into two parts. In the first part, considered as a screening one, a set of $\mathrm{n}=76$ samples of paraffin-embedded specimen of surgically removed metastatic brain tumors of NSCLC pa- tients only was analyzed (surgeries performed between 2005 and 2010). Median patient's age for this part of the study was 59.2 years and the female to male ratio was 21:55. Due to the high clinical heterogeneity of this group, at this stage the tumors were only characterized by the histological type: adenocarcinoma ( $\mathrm{AC}, \mathrm{n}=34$ ), squamous cell carcinoma ( $\mathrm{SCC}, \mathrm{n}=18$ ) and poorly differentiated large cell carcinoma (LCC, $\mathrm{n}=24$ ). Additional immunohistochemical staining with CK7 (cytokeratin 7) and TTF-1 (thyroid transcription factor 1) was performed if serious clinical considerations over the primary tumor origin existed. This also aided the differential diagnosis between LCC and poorly differentiated $\mathrm{AC}$ in the primary tumor assessment.

The second part of the study which aimed to answer whether and how $\alpha-2,6-$ GalSA presence in the primary tumor affected the prognosis involved a subset of patients from the first part of the study who met more strict inclusion criteria. Included were $n=20$ patients from the first part of the study, who: 1) had their primary tumor specimen available for analysis, 2) had their treatment (either surgery or definite radiochemotherapy) definitely completed, 3) had their full follow-up data available, 4) in whom the brain metastasis occurred no sooner than 4 months (median: 12.6) following surgery, and 5) in whom the brain was the first site of disease recurrence. The intensity of $\alpha-2,6-\mathrm{GalSA}$ staining in the primary tumor specimen (Figure $2 \mathrm{~A}-2 \mathrm{C}$ ) was correlated with the clinical outcome measured as overall (OS) and disease-free survival (DFS) time. In addition, an identical staining for $\alpha$-2,6-GalSA was performed on primary lung tumors of a control group of $\mathrm{n}=22$ patients who underwent curative surgery for NSCLC in the years 2004 and 2005 and who had no recurrence within 5 years of follow-up. Both groups are clinically characterized in Table 2 . It must be noted, that for logistic reasons related to the availability of tumor specimen, the case and control groups significantly varied by treatment modality and tumor nodal involvement. 
This could affect the interpretation of results relating to the comparison of these groups.

Statistical analysis. All statistical analysis was performed using Statistica 10 software (Statsoft Inc., Tulsa, OK, USA). Confidence level was set at $95 \%$; observations with p-values $<0.05$ were considered statistically significant. Parametric tests: Student's t-test, Spearman's correlations, ANOVA (analysis of variance) and Tukey post-hoc test were used for comparison of continuous values (i.e. the HC score), whereas for their gradual counterparts (i.e. positive or negative $\alpha$-2,6-GalSA expression status as described in Methods) the non-parametric Mann-Whitney U-test was used. The impact of $\alpha-2,6-\mathrm{GalSA}$ expression on survival was analyzed in Kaplan-Meier and multivariate Cox models.

\section{Results}

\section{$\alpha-2,6-G a l S A$ in different tumor types}

The tumors that were analyzed in both parts of the study showed a statistically significant difference in $\alpha$-2,6-GalSA expression as measured by SNA reactivity according to the tumor type. In the first, screening part of the study, $\alpha$-2,6-GalSA staining was found to be statistically stronger in adenocarcinoma (AC) and squamous cell carcinoma (SCC) than poorly differentiated large cell carcinoma (LCC) tumors (Table 3). No significant differences were noted between AC and SCC. A corresponding analysis in the primary tumors in the second part of the study (Table 4) also showed statistically significant differences, however, the $\alpha-2,6$-GalSA staining in AC was significantly stronger than in SCC and LCC whereas in SCC and LCC the levels were similar. The differences between tumor types were also significant when analyzed separately in case and control groups (as indicated in Table 5).

$\alpha$-2,6-GalSA expression in primary and metastatic tumor pairs. The aim of this analysis was to investigate whether $\alpha$-2,6-GalSA was up-regulated in the metastatic tumors compared to the primary ones, similarly to the situation reported in breast cancer [13]. Out of 20 metastatic brain tumors in our study, only 5 had different $\alpha-2,6-$ GalSA expression status (positive or negative) than their matched primary tumor. Average $\mathrm{HC}$ scores and the number of tumors positive for $\alpha-2,6-\mathrm{GalSA}$ by each tumor type are shown in Table 6 . The differences observed were not statistically significant, which justifies a conclusion that the expression of $\alpha-2,6-\mathrm{GalSA}$ in NSCLC remains constitutive during its clinical course.
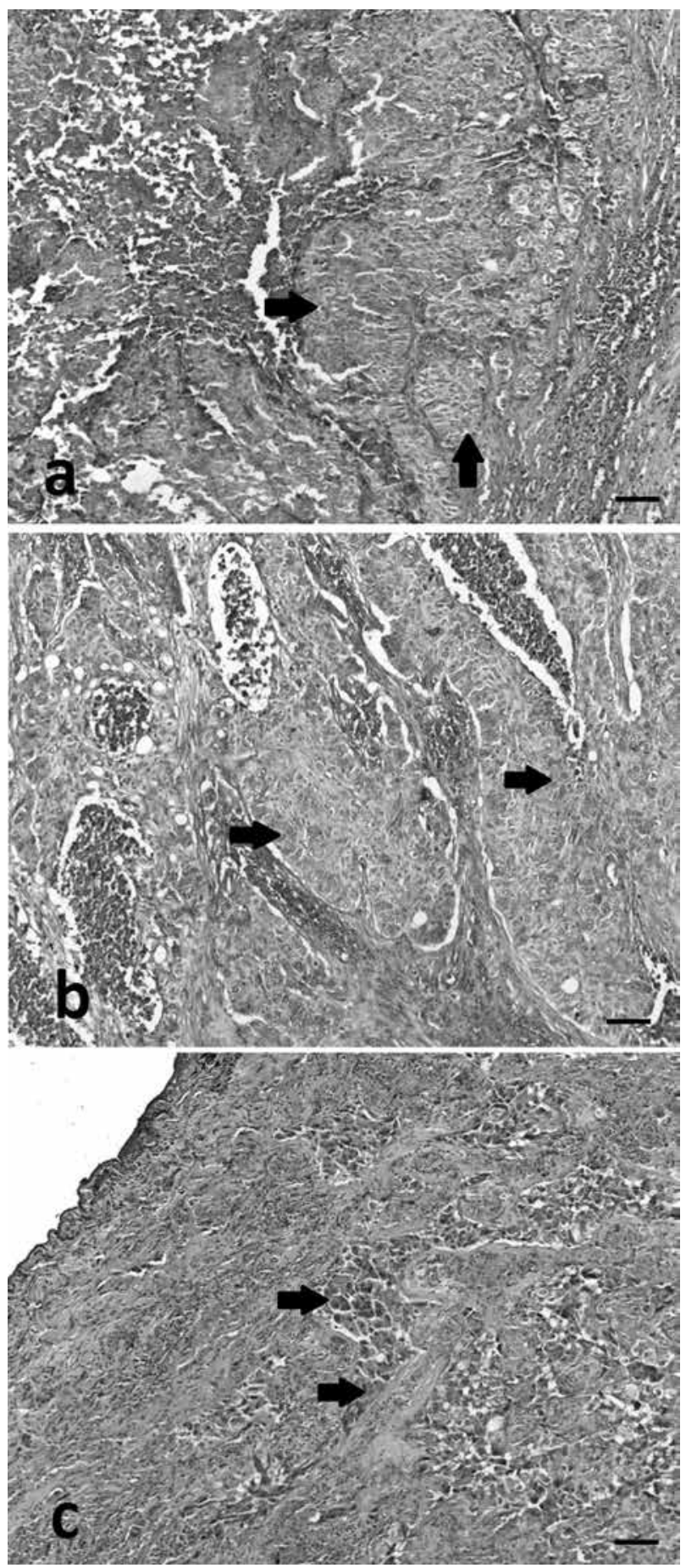

Figure 2. Representative examples of histochemical staining of $\alpha$-2,6-galactose-linked sialic acids by Sambucus nigra agglutinin (SNA). Tumor cells foci are indicated with black arrows. A. Squamous cell carcinoma of the lung: 0/+ staining intensity, HC score $1+1=2$. B. Squamous cell carcinoma of the lung: $(++)$ staining intensity, $\mathrm{HC}$ score $2+2=4$. Notice the high heterogeneity and dispersion of the SNA staining. C. Adenocarcinoma of the lung: $(+++)$ staining intensity, HC score $4+3=7$. Nuclei were counterstained with hematoxylin. Magnification $\times 100$; scale bars, $50 \mu \mathrm{m}$ 
Table 2. Clinical characteristics of case and control patient groups

\begin{tabular}{|c|c|c|c|}
\hline & Case group $(n=20)$ & Control group $(n=22)$ & Difference* \\
\hline Median patient age (years) & 59.3 & 61.8 & NS \\
\hline Patient sex (female/male) & $5 / 15$ & $4 / 18$ & NS \\
\hline $\begin{array}{l}\text { Tumor type } \\
\text { Adenocarcinoma (AC) } \\
\text { Squamous cell carcinoma (SCC) } \\
\text { Large cell carcinoma (LCC) }\end{array}$ & $\begin{array}{l}9 / 20 \\
4 / 20 \\
7 / 20\end{array}$ & $\begin{array}{l}9 / 22 \\
8 / 22 \\
5 / 22\end{array}$ & NS \\
\hline $\begin{array}{l}\text { Tumor grade } \\
\text { Low (G1-G2) } \\
\text { High (G3) } \\
\text { Not specified }\end{array}$ & $\begin{array}{c}6 / 20 \\
10 / 20 \\
4 / 20\end{array}$ & $\begin{array}{c}11 / 22 \\
10 / 22 \\
1 / 22\end{array}$ & NS \\
\hline Tumor nodal involvement (N1 or N2) & $7 / 20$ & $1 / 22$ & $\mathrm{P}=0.014$ \\
\hline $\begin{array}{l}\text { Treatment modality } \\
\text { Surgery } \\
\text { Definite chemoradiotherapy }\end{array}$ & $\begin{array}{l}10 / 20 \\
10 / 20\end{array}$ & $\begin{array}{c}22 / 22 \\
0 / 22\end{array}$ & $\mathrm{P}=0.00018$ \\
\hline
\end{tabular}

* Refers to the difference in proportion of a given clinical variable between case and control group; N1 — confirmed metastasis in hilar lymph nodes only, $\mathrm{N} 2$ - confirmed metastasis in ypsilateral mediastinal lymph nodes; NS — not significant

Table 3. Results of $\alpha-2,6-$ GalSA expression measured by histochemical staining in the metastatic brain tumors

\begin{tabular}{|l|c|c|c|c|c|}
\hline & \multirow{2}{*}{ Overall } & \multicolumn{3}{|c|}{ By tumor type } & \multirow{2}{*}{ Difference* } \\
\cline { 3 - 6 } & & AC & SCC & LCC & \\
\hline No. of tumors positive for $\alpha$-2,6-GalSA staining (\%) & $44 / 76$ & $24 / 34$ & $11 / 18$ & $9 / 24$ & $\mathrm{P}=0.004$ \\
& $57.9 \%$ & $70.5 \%$ & $61.1 \%$ & $37.5 \%$ & \\
\hline Average HC score & 5.26 & 5.82 & 5.44 & 4.33 & $\mathrm{P}=0.006$ \\
\hline
\end{tabular}

*Refers to the difference in $\alpha-2,6-$ GalSA expression between tumor types (most significant between AC and LCC, as determined by Tukey post-hoc test); abbreviations as described for Table 2, $\mathrm{HC}$ - histochemical

Table 4. Results of $\alpha-2,6-$ GalSA expression measured by histochemical staining in the primary lung tumors

\begin{tabular}{|l|c|c|c|c|c|}
\hline & \multirow{2}{*}{ Overall } & \multicolumn{3}{|c|}{ By tumor type } & \multirow{2}{*}{ Difference* } \\
\cline { 3 - 5 } & & AC & SCC & LCC & \\
\hline No. of tumors positive for $\alpha$-2,6-GalSA staining $(\%)$ & $31 / 42$ & $18 / 18$ & $6 / 12$ & $7 / 12$ & $\mathrm{P}=0.004$ \\
& $73.8 \%$ & $100 \%$ & $50 \%$ & $58.3 \%$ & \\
\hline Average HC score & 5.95 & 6.83 & 5.17 & 5.42 & $\mathrm{P}=0.002$ \\
\hline
\end{tabular}

* Refers to the difference in $\alpha-2,6$-GalSA expression between tumor types (most significant between AC and SCC, as determined by Tukey post-hoc test); abbreviations as described for Table 2

The prognostic value of $\alpha$-2,6-GalSA expression. A comparative analysis of $\alpha-2,6-\mathrm{GalSA}$ expression between case and control group (bearing in mind the previously described clinical differences between them) revealed some trends. While for AC the $\alpha$-2,6-GalSA staining was positive in all patients in both groups, SCC and LCC demonstrated a tendency towards less intense $\alpha-2,6$-GalSA staining in the case group than in the control one. However, the differences observed were not statistically significant (Table 5).
The analysis of survival times of patients from the case group partially confirmed this tendency. The analysis of the histochemical score (HC) in the Cox proportional hazard model found it to be significantly prognostic for OS (HR $=-0.4, \chi^{2}=4.4, \mathrm{p}=0.03 ; \mathrm{HR}=$ hazard ratio), but not for DFS $\left(\mathrm{HR}=-0.28, \chi^{2}=3.0, \mathrm{p}=0.08\right)$. In the Kaplan-Meier model, the observed median survival times also suggested a slightly better prognosis for the sub-group with high $\alpha$-2,6-GalSA HC scores, but in this model no statistical signifi- 
Table 5. Alpha-2,6-GalSA expression measured by histochemical staining in primary lung tumors in patients with (case group) and without (control group) brain metastases

\begin{tabular}{|c|c|c|c|c|c|}
\hline & \multicolumn{2}{|l|}{ Case } & \multicolumn{2}{|l|}{ Control } & \multirow[t]{2}{*}{ Difference* } \\
\hline & No. of positive cases (\%) & HC score & No. of positive cases (\%) & HC score & \\
\hline Overall & $13 / 20(65 \%)$ & 5.55 & $18 / 22(81.8 \%)$ & 6.32 & $\mathrm{NS}(\mathrm{P}=0.08)$ \\
\hline $\mathrm{AC}$ & 9/9 (100\%) & 6.67 & 9/9 (100\%) & 7.0 & $\mathrm{NS}(\mathrm{P}=0.06)$ \\
\hline SCC & $1 / 4(25 \%)$ & 4.25 & $5 / 8(62.5 \%)$ & 5.62 & $\mathrm{NS}(\mathrm{P}=0.21)$ \\
\hline LCC & $3 / 7(42.8 \%)$ & 4.85 & $4 / 5(80 \%)$ & 6.2 & $\mathrm{NS}(\mathrm{P}=0.13)$ \\
\hline Difference** & $\mathrm{P}=0.011$ & $\mathrm{P}=0.014$ & $P=0.03$ & $\mathrm{P}=0.032$ & \\
\hline
\end{tabular}

*Refers to the difference in $\alpha-2,6$-GalSA expression in tumors of a given type between case and control groups; ** refers to the difference in $\alpha$-2,6-GalSA expression between tumors of different types in case and control groups; abbreviations as described for Table 2

Table 6. Alpha-2,6-GalSA expression measured by histochemical staining in paired primary and metastatic tumors

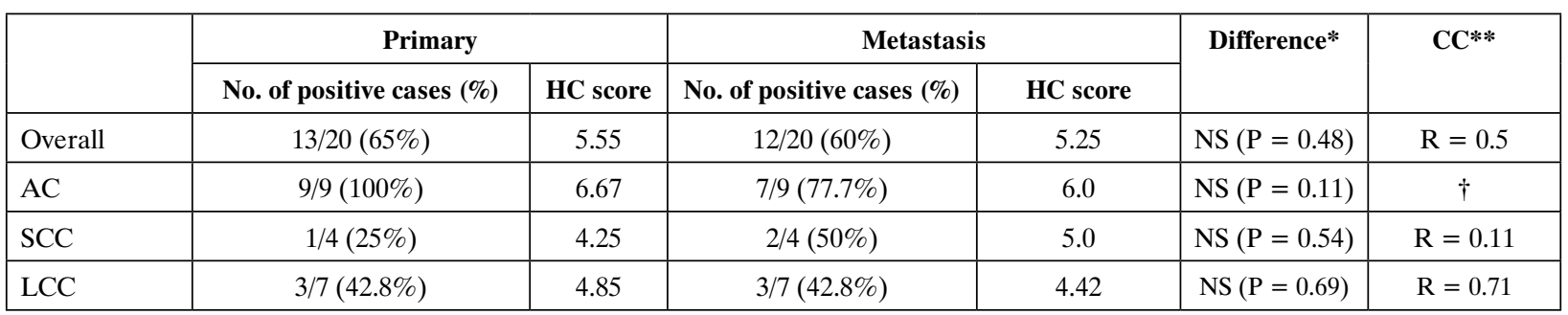

* Refers to the difference in $\alpha$-2,6-GalSA expression in tumors of a given type between primary and metastatic tumor groups; ** refers to the correlation between the expression of $\alpha-2,6-$ GalSA in matched primary metastatic and primary tumor pairs of a given type; $\dagger$ the calculated $\mathrm{R}$-value of $-1.5 \times 10^{-16}$ was not interpreted in the results; abbreviations as described for Table $2, \mathrm{CC}-$ correlation coefficient

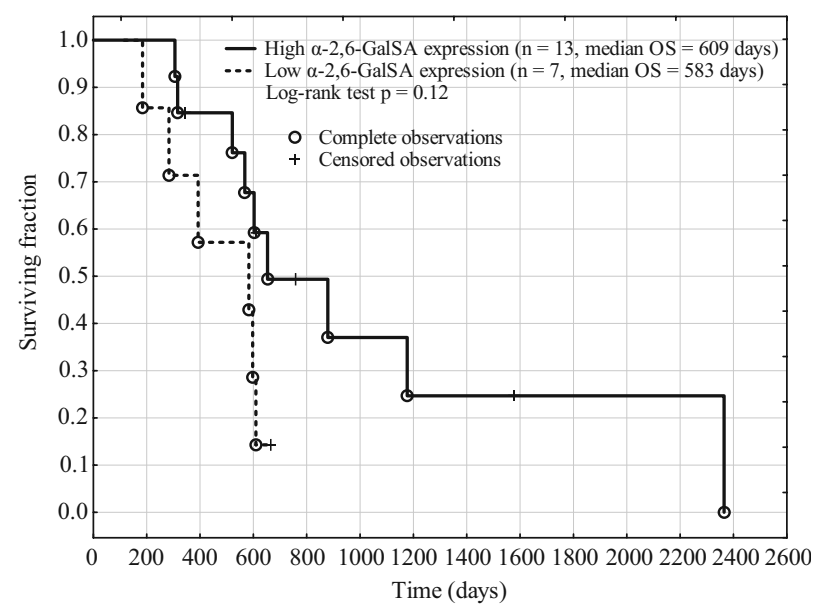

Figure 3. Kaplan-Meier curves for overall survival (OS) according to $\alpha$-2,6-galactose-linked sialic acids expression status in NSCLC cancer patients with brain metastases (control group without brain metastases was excluded)

cance for both OS and DFS was found (Figure 3 and 4). Patients' age, sex, tumor type, tumor grade, nodal status and treatment modality were not identified as significant survival predictors in either of the survival estimation models within the group of NSCLC patients with BM.

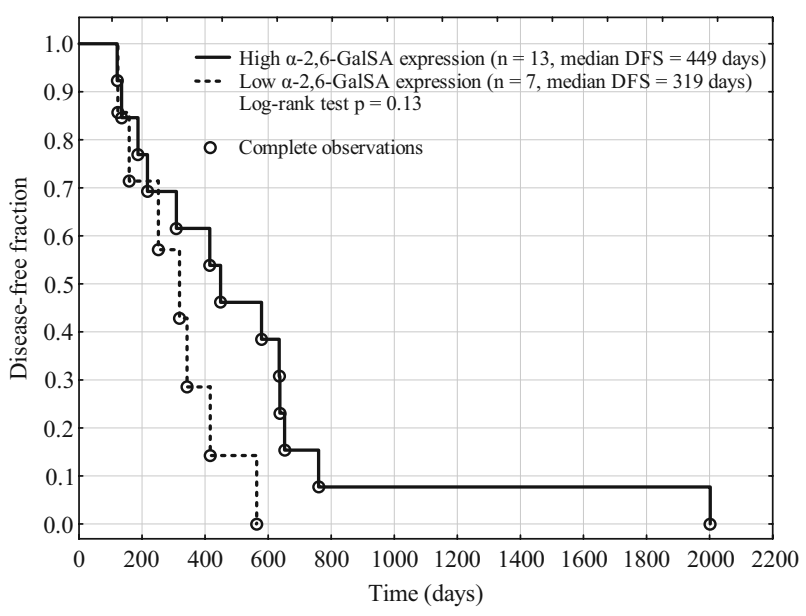

Figure 4. Kaplan-Meier curves for disease-free survival (DFS) according to $\alpha$-2,6-galactose-linked sialic acids expression status in NSCLC patients with brain metastases (control group without brain metastases was excluded)

\section{Discussion}

The presented study on the detection by histochemical staining of $\alpha$-2,6-galactose-linked sialic acids in nonsmall-cell lung cancer and in its brain metastases is the 
first attempt to study the presence of these compounds in this tumor type. Our study reported no changes of the $\alpha-2,6-$ GalSA presence status, defined by histochemical staining reaction, between primary and metastatic tumors. Alpha-2,6-GalSA expression, whenever high or low, remained stable in matched tumor pairs. This finding is in contrast to the results of breast cancer study in which the overexpression of ST6GalNAC5 and higher SNA reactivity in brain metastasis compared to the primary breast tumor was reported [13]. However, when referred to the clinical characteristics of breast and lung cancer, this discrepancy may seem logical. Metastases of breast cancer to the brain typically take years to develop and are relatively rare, in particular among those patients who have been successfully managed locally [18]. In such clinical circumstances, BM could possibly develop from selected, aggressive single cell clones which gain metastatic potential over time. On the contrary, lung cancer is a more aggressive tumor which spawns brain metastases much more frequently and relatively sooner - it is therefore predictable that the metastatic potential of the latter is either acquired sooner than in breast cancer or initially present, as the result of our study may suggest.

Similar contradictions arise with an attempt to answer - using the results of this study - the key question: can $\alpha-2,6-\mathrm{GalSA}$ presence be a predictor of brain metastasis originating from the lung cancer? Discretely lower expression of $\alpha-2,6-\mathrm{GalSA}$ was observed in the case group compared to the control one. However, this result should be taken with caution due to the significant differences in tumor stage and treatment modalities - this preliminary finding needs validation in further studies with more uniform patients' characteristics. However, within the case group only, the overall and disease-free survival times were longer for patients with tumors that presented higher $\alpha-2,6-\mathrm{GalSA}$ expression. This result is in line with other studies which reported down-regulation of $\alpha-2,6-\mathrm{GalSA}$ in metastatic brain tumors [19]. The underlying mechanisms could be explained by the functioning of $\alpha-2,6$-GalSA not only as mediating adhesion of tumor cells to the vascular endothelium [13] but also acting as adhesion molecules between cells of same type [20]. An impaired adhesion of cells within the tumor would therefore likely enable their release into blood or lymphatic vessels and their migration to the brain (and other organs) with $\alpha-2,6-\mathrm{GalSA}$ compromising then blood-brain barrier [13].

As already mentioned, the prognostic value of $\alpha-2,6-$ GalSA presence in various tumors and their correlation with clinical variables remains still to be established, especially given the contradictory re- ports from available studies. Some authors reported the higher immunoreactivity of the enzyme $\alpha-2,6-$ sialyltransferase in well differentiated tumors (e.g. in hepatic cell carcinoma [21]), but others found the $\alpha$-2,6-GalST immunoexpression to be more prevalent in tumors of higher histological grade (colorectal cancer [11], pancreatic cancer cell line [12]). The same concerns the influence of on cellular motility and invasive capabilities. For particular cell types overexpression of $\alpha$-2,6-GalSA or $\alpha$-2,6-ST enzyme has been associated with enhanced tumor migration potential (breast cancer cell line [22], colon cancer cell lines [23], HCC cell line [24]), whereas for other cell types this phenomenon results in impaired departure from the originating tissue (lymphosarcoma cell line MDAY-D2 [25]) and inhibition of tumor growth in vivo (glioma [26]).

The role of $\alpha-2,6-$ GalSA and $\alpha-2,6-\mathrm{ST}$ in NSCLC cannot be unanimously defined using the data from this preliminary study mostly due to inadequate size of the group studied. The obtained results suggest that NSCLC rather falls into the category of tumors in which the high expression of $\alpha$-2,6-GalSA indicates a better prognosis, or is at least more frequently expressed in higher differentiated tumors which are commonly associated with a more favorable outcome. Lack of differences in $\alpha$-2,6-GalSA expression between primary NSCLC tumors and brain metastases derived from those, when related to the contrary observation of the study on $\alpha-2,6-\mathrm{GalSA}$ in breast cancer brain metastases [13] indicates that the mechanisms responsible for brain metastasis formation in these tumor types can be different and either absent in the primary tumor and acquired subsequently by circulating tumor cells (as in breast cancer) or initially present and constitutive during the clinical course (NSCLC). An experimental in vivo study on the results of the knockout of ST6GalNAC gene in the tumor cell lines which overexpress $\alpha$-2,6-GalSA on the in vivo tumor growth could clarify the role of this type of sialic acids in various tumor types, including NSCLC, and the mechanism of their dissemination into the brain.

\section{Acknowledgements}

We would like to thank the following individuals who by their physical, organizational and financial effort voluntarily contributed to this study: Violetta Bryda, Jolanta Kijek, Barbara Nikiel, Iwona Pasnik, Bozena Pelak, Dariusz Pelak, Marek Sawicki, Kamila Wojas-Krawczyk. This study was funded with an internal grant by Medical University of Lublin and first author's own resources. 


\section{References}

1. GLOBOCAN 2012: Estimated cancer incidence, mortality and prevalence worldwide in 2012. Available from URL: http://globocan.iarc.fr. Viewed: 10th Jan 2014.

2. Komaki R, Scott CB, Byhardt R et al. Failure patterns by prognostic group determined by recursive partitioning analysis (RPA) of 1547 patients on four radiation therapy oncology group (RTOG) studies in inoperable non-small-cell lung cancer (NSCLC). Int J Radiat Oncol Biol Phys. 1998;42:263-267.

3. Yaeger KA, Nair MN. Surgery for brain metastases. Surg Neurol Int. 2013;4:203.

4. Milas I, Komaki R, Hachiya T et al. Epidermal growth factor receptor, cyclooxygenase-2, and bax expression in the primary non-small cell lung cancer and brain metastases. Clin Cancer Res. 2003;9:1070-1076.

5. D'Amico T, Aloia T, Moore M-B et al. Predicting the sites of metastases from lung cancer using molecular biologic markers. Ann Thorac Surg. 2001;72:1144-1148.

6. Arnold S, Young A, Munn R et al. Expression of p53, bcl-2, e-cadherin, matrix metalloproteinase- 9 , and tissue inhibitor of metalloproteinases- 1 in paired primary tumors and brain metastasis. Clin Cancer Res. 1999;5:4028-4033.

7. Taatjes DJ, Roth J. Alteration in sialyltransferases and sialic acid expression accompanies cell differentiation in rat intestine. Eur J Cell Biol. 1988;46:289-298.

8. Hakomori SI. Bifunctional role of glycolipids. Modulators for transmembrane signalling and mediators for cellular interactions. J Biol Chem. 1990;265:18 713-18 716.

9. Powell LD, Whiteheart SW, Hart GW. Cell surface sialic acid influences tumor cell recognition in the mixed lymphocyte reaction. J Immunol. 1987;139:262-270.

10. Wang PH. Altered glycosylation in cancer: Sialic acids and sialyltransferases. J Cancer Molec. 2005;1:73-81.

11. Lise M, Belluco C, Perera SP, Patel R, Thomas P, Ganguly A. Clinical correlations of alpha-2,6-sialyltransferase expression in colorectal cancer patients. Hybridoma. 2000;19:281-286.

12. Perez-Garay M, Arteta B, Pages L et al. $\alpha 2,3$-sialyltransferase ST3Gal III modulates pancreatic cancer cell motility and adhesion in vitro and enhances its metastatic potential in vivo. PLoS One. 2010;5:e12524. doi:10.1371/jounal.pone.0012524.

13. De Bos $\mathrm{P}, \mathrm{Zhang} X, \mathrm{Nadal} C$ et al. Genes that mediate breast cancer metastasis to the brain. Nature. 2009;459:1005-1009.

14. Chiang $\mathrm{CH}$, Wang $\mathrm{CH}$, Chang HC, More S, Li WS, Hung WC. A novel sialyltransferase inhibitor AL10 suppresses invasion and metastasis of lung cancer cells by inhibiting integrin-mediated signaling. J Cell Physiol. 2010;223:492$-499$.

15. Varki A, Cummings RD, Esko JD et al. Essentials of Glycobiology (2nd edition). Cold Spring Harbor (NY); Cold Spring Harbor Laboratory Press 2009.

16. Kaneko Y, Hirotaka Y, Colley KJ, Moskal J. Expression of $\mathrm{Gal} \beta 1,4 \mathrm{GlcNAc} \alpha$-2,6-sialyltransferase and $\alpha$-2,6-linked sialoglycoconjugates in normal human and rat tissues.J Histochem Cytochem. 1995;43:945-954.

17. Harvey JM, Clark GM, Osborne CK, Allred DC. Estrogen receptor status by immunohistochemistry is superior to the ligand-binding assay for predicting response to adjuvant endocrine therapy in breast cancer.J Clin Oncol. 1999;17:1474-1481.

18. Kennecke H, Yerushalmi R, Woods R et al. Metastatic behavior of breast cancer subtypes.J Clin Oncol. 2010;28:3271$-3277$.

19. Kaneko Y, Yamamoto H, Kersey DS, Colley KJ, Leestma JE, Moskal JR. The expression of Gal beta 1,4GlcNAc alpha 2,6 sialyltransferase and alpha 2,6-linked sialoglycoconjugates in human brain tumors. Acta Neuropathol. 1996;91:284-292.

20. Barnes YC, Skelton TP, Stamenkovic I, Sgroi D. Sialylation of the sialic acid binding lectin sialoadhesin regulates its ability to mediate cell adhesion. Blood. 1999;93:1245-1252.

21. Poon T, Chiu C, Lai P et al. Correlation and prognostic significance of beta-galactoside alpha-2,6-sialyltransferase and serum monosialylated alpha-fetoprotein in hepatocellular carcinoma. World J Gastroenterol. 2005;11:6701-6706.

22. Lin S, Kemmner W, Grigull S, Schlag PM. Cell surface alpha2,6-sialylation affects adhesion of breast carcinoma cells. Exp Cell Res. 2002;276:101-110.

23. Matsushita Y, Hoff SD, Nudelman ED. Metastatic behavior and cell surface properties of HT-29 human colon carcinoma variant cells selected for their differential expression of sialyl-dimeric Le(x)-antigen. Clin Exp Metastasis. 1991;9:283$-299$.

24. Yu S, Fan J, Liu L, Zhang L, Wang S, Zhang J. Caveolin-1 up-regulates integrin $\alpha 2,6$-sialylation to promote integrin $\alpha 5 \beta 1$-dependent hepatocarcinoma cell adhesion. FEBS Lett. 2013;587:782-787.

25. Dennis J, Waller C, Timpl R, Schirrmacher V. Surface sialic acid reduces attachment of metastatic tumour cells to collagen type IV and fibronectin. Nature. 1982;300:274-276.

26. Kroes RA, He H, Emmett MR et al. Overexpression of ST6GalNAcV, a ganglioside-specific alpha-2,6-sialyltransferase, inhibits glioma growth in vivo. Proc Natl Acad Sci USA. 2010;107:12 646-12 651. 